\title{
Artemther-lumefantrine is partially effective for treating chronic multi-species malaria in Ugandan pre-school children
}

\author{
Martha Betson ${ }^{1 *}$, José C Sousa-Figueiredo ${ }^{1,2}$, Sarah Clifford ${ }^{1}$, Aaron Atuhaire ${ }^{3}$, Moses Arinaitwe ${ }^{3}$, Moses Adriko ${ }^{3}$, \\ Gerald Mwesigwa $^{3}$, Juma Nabonge ${ }^{4}$, Narcis B Kabatereine ${ }^{3}$, Colin J Sutherland ${ }^{2}$, J Russell Stothard ${ }^{1}$
}

From Challenges in malaria research

Basel, Switzerland. 10-12 October 2012

\section{Background}

In Uganda artemether-lumefantrine $(\mathrm{AL})$ is the first-line treatment for uncomplicated falciparum malaria. During a longitudinal study (SIMI project) investigating the dynamics of intestinal schistosomiasis and malaria in Ugandan lakeshore communities, a consistently high prevalence of malaria was found in young children (including many mixed infections of Plasmodium falciparum with P. malariae and/or P. ovale sspp), despite use of AL for home-based management of malaria. As a first step to gather evidence on the effectiveness of AL in this setting, a community-based observational study was initiated in an area of intense malaria transmission.

\section{Materials and methods}

The study was carried out in Bukoba village, Mayuge District. A group of children $(\mathrm{W}=163)$ within the SIMI cohort was selected on the basis of a positive First Response rapid diagnostic test (RDT) result and microscopy-confirmed malaria. Children were actively followed up 7 and 17 days after baseline. Individuals who were RDT-positive on Day 17 were also followed up on Day 24. At each time-point, a blood smear archive was made and microscopy was performed two-three days after samples were taken. In addition, blood spots were collected onto Whatmann 3MM filter paper. Treatment decisions in the field were based on RDT results. All study participants were treated with AL at baseline. Children who were malaria positive by OptiMAL RDT on Day 7 were retreated with AL and those who were malaria positive on Day 17 were treated with oral quinine. All children were

'Liverpool School of Tropical Medicine, Liverpool L3 5QA, UK Full list of author information is available at the end of the article tested for Schistosoma mansoni and soil-transmitted helminth infections at baseline and treated with praziquantel and albendazole.

Genomic DNA was extracted from blood spots using chelex and real-time PCR diagnosis of Plasmodium species was performed. To distinguish between recrudescent and new infections, genotyping of merozoite surface proteins ( $m s p 1$ and $m s p 2$ ) and glutamate-rich protein ( glurp) was carried out.

\section{Results}

Forty children (26.3\%) were microscopy-positive for malaria on Day 7 and 33 (21.3\%) on Day 17. After genotyping, 33 (21.9\%) and 17 (11.7\%) children were shown to have recrudescent infections on Days 7 and 17, respectively. Of the 28 children who had received two consecutive AL treatments, 11 were microscopy positive on Day 17.

Multi-species Plasmodium infections were common, with $41.1 \%$ of children positive for $P$. falciparum/ P. malariae, $9.2 \%$ positive for $P$. falciparum/P. ovale sspp. and $8.0 \%$ for all three species at baseline. By realtime PCR $39.9 \%$ of those children infected with falciparum malaria at baseline were $P$. falciparum positive at Day 17 and $9.2 \%$ of those who were infected with $P$. malariae at baseline were $P$. malariae positive at Day 17. On Day 24, after two or three consecutive antimalarial treatments, 10 children were infected with $P$. falciparum and two with $P$. malariae.

\section{Conclusions}

Our results suggest that AL may not be as effective as previously thought for treatment of malaria at a communitybased level in Uganda and that further more formalised 
efficacy studies of this drug in high transmission settings are required, particularly in areas where mixed-species malaria infections are common and where mass administration of anthelminthic drugs is being carried out.

\section{Author details}

'Liverpool School of Tropical Medicine, Liverpool L3 5QA, UK. ${ }^{2}$ Faculty of Infectious and Tropical Diseases, London School of Hygiene and Tropical Medicine, London WCIE 6BT, UK. ${ }^{3}$ Vector Control Division, Ministry of Health, Kampala, Uganda. ${ }^{4}$ Vector Control Division, Mayuge District, Mayuge,

Uganda.

Published: 15 October 2012

doi:10.1186/1475-2875-11-S1-P11

Cite this article as: Betson et al:: Artemther-lumefantrine is partially effective for treating chronic multi-species malaria in Ugandan preschool children. Malaria Journal 2012 11(Suppl 1):P11.

Submit your next manuscript to BioMed Central and take full advantage of:

- Convenient online submission

- Thorough peer review

- No space constraints or color figure charges

- Immediate publication on acceptance

- Inclusion in PubMed, CAS, Scopus and Google Scholar

- Research which is freely available for redistribution

Submit your manuscript at www.biomedcentral.com/submit 